\title{
POLYGONAL PRODUCTS OF POLYCYCLIC BY FINITE GROUPS
}

\author{
R.B.J.T. Allenby
}

\begin{abstract}
We prove that a polygonal product of polycyclic by finite groups amalgamating normal subgroups, with trivial mutual intersections, is cyclic subgroup separable. Because of a recent example (stated below) of the author this substantial improvement on a recent theorem of Kim is essentially best possible.
\end{abstract}

\section{INTRODUCTION}

Over the last few years interest has been shown in studying the structure, in particular the residual structure, of so called polygonal products of groups which are defined as follows.

Definitions and Notation: Let $\mathcal{P}$ be a polygon. To each vertex, $\nu$, of $\mathcal{P}$ assign a "vertex group" $A_{\nu}$ and to each edge, $e$, of $\mathcal{P}$ assign an "edge group" $A_{e}$ and a pair $\lambda_{e}$, $\mu_{e}$ of monorphisms embedding $A_{e}$ as a subgroup of each of the two vertex groups at the ends of $e$. The polygonal product of this system of groups is the group $P$ with generators and relations those of the vertex groups together with the extra relations obtained by identifying $a_{e} \lambda_{e}$ and $a_{e} \mu_{e}$ for each $a_{e}$ in $A_{e}$. Note that, if the polygon is a triangle then $P$ may not contain the vertex groups isomorphically. (See [9].) As a consequence we shall consider here only the case where the polygon has four edges, the cases of polygons with more than four edges being just as readily dealt with. Denoting the vertex groups (initially) by $A_{i}(1 \leqslant i \leqslant 4)$ and the embedded subgroups $A_{i} \cap A_{i+1}$ by $K_{i}$ (all suffices being taken modulo 4), we shall assume throughout that each $K_{i} \cap K_{i+1}$ is trivial. Then $P$ is a generalised free product, $P=\left(A_{1} *_{K_{1}} A_{2}\right) *_{K_{2} * K_{4}}\left(A_{3} * K_{3} A_{4}\right)$ \{of generalised free products $\}$ - so that $P$ contains all the $A_{i}$ and their intersections isomorphically. (This construction was used very effectively by Higman [6] in his construction of the first example of a finitely generated infinite simple group.)

We shall use $g p\{\ldots\}$ to denote the subgroup generated by the subgroups inside the brackets, and $H \triangleleft G\left(H \triangleleft_{f} G\right)$ to denote that $H$ is a normal subgroup (normal subgroup of finite index) in $G$.

A cyclic subgroup of a group $G$ which is contained in no larger cyclic subgroup of $G$ is called a maximal cyclic subgroup of $G$. (See Example B below.)

Received 6 December 1995

Copyright Clearance Centre, Inc. Serial-fee code: 0004-9729/96 \$A2.00+0.00. 
For our main result we shall need the following definition:

Definttion: Let $G$ be a group and $H$ a subgroup of $G . G$ is said to be $H$ separable if and only if for each element $g \in G \backslash B$ there exists a finite homomorphic image $\bar{G}$ of $G$ for which $\bar{g} \notin \bar{H}$. If $G$ is $H$-separable for all finitely generated subgroups $H$ of $G$ then $G$ is called subgroup separable (sometimes LERF) whilst if $G$ is $H$ separable for all cyclic subgroups $H$ of $G$ then $G$ is called cyclic subgroup separable (or, quite often, $\pi_{c}[\mathbf{1 0}]$.)

(Thus all such groups are quite strongly residually finite.)

Recently, Goansu Kim [7] has greatly extended one of the early (and rather trivial) results (see [3]) concerning the residual finiteness of a polygonal product of four finitely generated free Abelian groups amalgamating cyclic subgroups (and, of course, with trivial intersections) by proving:

THEOREM A. (Kim [7].) Let $P$ be a polygonal product of four polycyclic by finite groups $A_{1}, A_{2}, A_{3}$ and $A_{4}$ where each amalgamated subgroup $K_{i}=A_{i} \cap A_{i+1}$ lies in the centres of $A_{i}$ and $A_{i+1}$. Then $P$ is $\pi_{c}$.

There certainly has to be some fairly strong condition on the $K_{i}$ because of the following result in [1].

EXAMPLE B. There exists a non-residually-finite polygonal product $P$ of four torsion free nilpotent groups $A_{1}, A_{2}, A_{3}$ and $A_{4}$ in which each $K_{i}=A_{i} \cap A_{i+1}$ is a maximal cyclic subgroup in each of $A_{i}$ and $A_{i+1}$.

The following result thus extends Kim's result just about as far as is possible.

THEOREM C. Let $P$ be a polygonal product of four polycyclic by finite groups $A_{1}, A_{2}, A_{3}$ and $A_{4}$ where each amalgamated subgroup $K_{i}=A_{i} \cap A_{i+1}$ is normal in each of $A_{i}$ and $A_{i+1}$ (and, for each $i, K_{i} \cap K_{i+1}=\langle 1\rangle$, the trivial subgroup). Then $P$ is $\pi_{c}$.

In proving the theorem we shall use the following result of $\mathrm{Kim}$ [8].

Lemma D. Let $G=E *_{H} F$. Suppose that:

(a) Both $E$ and $F$ are $\pi_{c}$ and $H$-separable;

(b) For each $N \triangleleft_{f} H$ there exist $N_{E} \triangleleft_{f} E$ and $N_{F} \triangleleft_{f} F$ such that $N_{E} \cap H=N_{F} \cap H \subseteq N$. Then $G$ is $\pi_{c}$.

Proof of Theorem C: We can now embark on the proof of Theorem C. We assume the conditions imposed by the statement of Theorem $\mathrm{C}$ but, for convenience, we replace $A_{1}, A_{2}, A_{3}, A_{4}$ and $K_{1}, K_{2}, K_{3}, K_{4}$ by $A, B, C, D$ and $U, K, V, H$ respectively.

SATISFYING KIM's CONDITIONS. We now recall that the polygonal product as given is just the generalised free product of $A *_{U} B$ and $D * V C$ with the free product 
$g p\{H, K\}=H * K$ amalgamated. We now show that Kim's Lemma, above, is again applicable. Since $A *_{U} B$ and $D *_{V} C$ are each subgroup separable (see [2, Theorem 5]) it is immediate that each group is $\pi_{c}$ and (since $H, K$ and hence $g\{H, K\}$ are finitely generated) $H * K$-separable. The latter shows that $A *_{U} B$ and $D *_{V} C$ satisfy Kim's condition (a) so we only need check that condition (b) of Kim's Lemma is satisfied.

To do this we prove

Lemma E. Let $A *_{U} B$ be as above and let $Z$ be a normal subgroup of finite index in $g p\{H, K\}=H * K \subseteq A *_{U} B$. Then there exists a subgroup $W$, which is contained in $Z$ and normal and of finite index in $H * K$ and which is completely determined by how $Z$ lies in $H * K$, being independent of how $Z$ lies in $A *_{U} B$, and a subgroup $Q$ of finite index in $A *_{U} B$ meeting $H * K$ precisely in $W$.

Proof: First pass from $A *_{U} B$ to the ordinary free product $S * T=A / U * B / U$ (recall that $U \triangleleft A$ and $U \triangleleft B$ ) - so that $H * K$ is preserved isomorphically. We retain the notation $H * K$ for this subgroup of $S * T$. Now in $S * T, g p\{H, K\}=H * K$, $g p\{S, K\}=S * K=S *_{H}\{H * K\}$ and $S * T=\left\{S *_{H}\{H * K\}\right\} *_{K} T$.

Now, $Z$ determines $Z \cap H$ and $Z \cap K$. Let $H_{0}$ and $K_{0}$ be subgroups, characteristic and of finite index in $H$ and $K$ and contained in $Z \cap H$ and $Z \cap K$ respectively. Thus $H_{0}$ and $K_{0}$ are normal in $S$ and $T$ respectively. Consider the natural homomorphism of $H * K$ onto $F=H / H_{0} * K / K_{0}$, a free product of finite groups. The kernel of this homomorphism is contained in $Z$ and meets $H$ and $K$ in $H_{0}$ and $K_{0}$ respectively. Further, the image of $Z$ in this homomorphism is a normal subgroup of finite index in $F$. Since $F$ is residually finite $[2,4,5]$ it is easy to find a normal subgroup $Z_{0}$ of finite index in $H * K$ such that $Z_{0} \subseteq Z$ and such that $Z_{0} \cap H=H_{0}$ and $Z_{0} \cap K=K_{0}$. (There are many possible choices for $Z_{0}$. Fix on any one.)

Note that $Z_{0}$ is totally determined within $H * K$. Its choice is independent of any larger subgroup of $S * T . \quad Z_{0}$ is the subgroup $W$ promised in the statement of the lemma.

We now show how to find the normal subgroup $Q$ of finite index in $A *_{U} B$ such that $Q \cap(H * K)=Z_{0}$.

In $S * K\left(=S *_{H}\{H * K\}\right)$ we have $Z_{0} \subseteq\{H * K\}$ and $Z_{0} \cap S=H_{0}$ - a normal subgroup of $S$. From the theory of generalised free products there is a homomorphism from $S *_{H}\{H * K\}$ onto $S / H_{0} *_{H / H_{0}}\{H * K\} / Z_{0}$ and then, since $S / H_{0}$ is polycyclic by finite and since $\{H * K\} / Z_{0}$ is finite, we see that $S / H_{0} *_{H / H_{0}}\{H * K\} / Z_{0}$ is residually finite $[2,4]$. It follows immediately that there is a normal subgroup of finite index in $S / H_{0} *_{H / H_{0}}\{H * K\} / Z_{0}$ which has trivial intersection with $\{H * K\} / Z_{0}$. The inverse image, $Z_{1}$, say, of this subgroup in $S *_{H}\{H * K\}$ thus meets $H * K$ in $Z_{0}$ exactly.

Finally we consider $S * T$ in the form $\left\{S *_{H}\{B * K\}\right\} *_{K} T$. The normal subgroup $Z_{1}$ of $S *_{H}\{H * K\}$ meets $T$ in $Z_{0} \cap K=K_{0}$, a normal subgroup of finite index in 
$T$. We may thus pass to the generalised free product $\left\{S *_{H}\{H * K\}\right\} / Z_{1} *_{K / K_{0}} T / K_{0}$ of finite groups. Since this group is residually finite $[2,4,5]$ we see that $S * T=$ $\left\{S *_{H}\{H * K\}\right\} *_{K} T$ has a normal subgroup of finite index meeting $S *_{H}\{H * K\}$ in precisely $Z_{1}$ and, hence, $H * K$ in precisely $Z_{0}$. The inverse image of this subgroup in $A *_{U} B$ is the subgroup $Q$ sought.

This completes the proof of Theorem C.

\title{
REFERENCES
}

[1] R.B.J.T. Allenby, 'The residual finiteness of polygonal products - two counterexamples', Canad. Math. Bull. 37 (1994), 433-436.

[2] R.B.J.T. Allenby and R.J. Gregorac, 'On locally extended residually finite groups', in Lecture Notes in Mathematics 319 (Springer-Verlag, Berlin, Heidelberg, New York, 1973), pp. 9-17.

[3] R.B.J.T. Allenby and C.Y. Tang, 'On the residual finiteness of certain polygonal products', Canad. Math. Bull. 32 (1989), 11-17.

[4] G. Baumslag, 'On the residual finiteness of generalized free products of nilpotent groups', Trans. Amer. Math. Soc. 106 (1963), 193-209.

[5] K.W. Gruenberg, 'Residual properties of infinite soluble groups', Proc. London Math. Soc. 7 (1957), 29-62.

[6] G. Higman, 'A finitely generated infinite simple group', J. London Math. Soc. 26 (1951), 61-64.

[7] Goansu Kim, 'On polygonal products of finitely generated Abelian groups', Bull. Austral. Math. Soc. 45 (1992), 453-462.

[8] Goansu Kim, 'Cyclic subgroup separability of generalized free products', Canad. Math. Bull. 36 (1993), 296-302.

[9] B.H. Neumann, 'An essay on free products of groups with amalgamations', Proc. Roy. Soc. Ser. A 246 (1954), 503-554.

[10] P. Stebe, 'Residual finiteness of a class of knot groups', Comm. Pure Appl. Math. 21 (1968), 563-583.

\author{
Department of Pure Mathematics \\ University of Leeds \\ Leeds LS2 9JT \\ United Kingdom \\ e-mail: PMT6RA@LEEDS.AC.UK
}

